EOMmun Communication et organisation

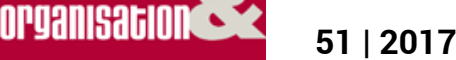

Les nouvelles cultures de l'information dans les organisations

\title{
Culture informationnelle collaborative et intelligence économique
}

Informational collaborative culture and economical intelligence

\section{Claire d'Hennezel}

\section{OpenEdition}

\section{Journals}

Édition électronique

URL : http://journals.openedition.org/communicationorganisation/5591

DOI : 10.4000/communicationorganisation.5591

ISBN : 979-10-300-0146-4

ISSN : 1775-3546

\section{Éditeur}

Presses universitaires de Bordeaux

\section{Édition imprimée}

Date de publication : 1 juin 2017

Pagination : 175-194

ISBN : 979-10-300-0162-4

ISSN : $1168-5549$

\section{Référence électronique}

Claire d'Hennezel, «Culture informationnelle collaborative et intelligence économique »,

Communication et organisation [En ligne], 51 | 2017, mis en ligne le 01 juin 2020, consulté le 01 janvier 2021. URL : http://journals.openedition.org/communicationorganisation/5591 ; DOI : https://doi.org/ 10.4000/communicationorganisation.5591 


\title{
Culture informationnelle collaborative et intelligence économique
}

\author{
Claire d'Hennezel'
}

Si l'on prend le rapport Martre ${ }^{2}$ (Martre 1994) comme référence, l'intelligence économique (IE) en France a aujourd'hui plus de 20 ans. En réalité les entreprises françaises s'étaient emparées de cette stratégie bien avant et les premières publications d'ouvrages professionnels datent des années 80 . L'intelligence économique présente la caractéristique originale d'être à la fois une stratégie d'entreprise, un objet scientifique et une politique publique. $\mathrm{Ce}$ caractère hybride lui confère un aspect composite qui en fait sa singularité, nourrit un certain nombre de controverses ou polémiques, en définitive, en fait un objet d'étude fécond et attractif.

Il existe une quantité notable de définitions de l'IE et à ce titre on peut se replonger dans le rapport Carayon (Carayon 2003), second rapport étatique sur le sujet, publié en 2003, qui ne recense pas moins de 22 définitions de ce terme " (Marcon 2014). Relevant le défi de "l'impossible définition " (Moinet 2011), nous entreprenons de définir ce concept et synthétiser les éléments qui nous semblent faire aujourd'hui consensus.

L'IE peut être définie comme une approche stratégique globale en vue d'accompagner l'entreprise dans ses actions à divers niveaux d'activité : la base, en interne ; les niveaux intermédiaires, locaux et enfin au niveau international (Martre 1994).

Cet ensemble d'actions s'effectue dans trois domaines principaux. Le premier, anticipateur, correspond à une série d'actions de collecte, d'analyse et de traitement de l'information utile, en vue d'améliorer le positionnement concurrentiel de l'entreprise dans son environnement. Le second, défensif, correspond à une démarche de sécurité économique visant à protéger le patrimoine de l'entreprise. Le troisième, proactif, consiste à développer une

1 Claire d'Hennezel est doctorante en SIC à l'Université Bordeaux Montaigne. Rattachée au laboratoire du MICA et son axe COS, Communication, Organisation et Société. Ses travaux portent sur l'intelligence économique et la veille, sur les petites entreprises en secteur innovant. Elle enseigne par ailleurs depuis de nombreuses années comme professeur certifiée en Economie et Gestion ; clairedhennezel@yahoo.fr

2 H. MARTRE, 1994, Intelligence économique et stratégie des entreprises. Paris: La Documentation Française. Premier rapport étatique comportant le terme intelligence économique dans son titre. 
démarche et des actions d'influence vis-à-vis de l'environnent, dans l'objectif " de peser sur les organisations qui établissent les règles juridiques et les normes internationales» (Martre 1994).

Enfin, depuis le rapport Carayon, l'intelligence économique est devenue une politique publique ayant comme dessein d'améliorer la " performance des entreprises en vue d'assurer la prospérité du territoire " grâce à "l'identification des secteurs et des technologies stratégiques et à l'organisation de la convergence des intérêts entre la sphère publique et la sphère privée, la puissance d'un pays ou d'un ensemble de pays partageant une communauté de destin » (Carayon 2003).

L'IE est donc un objet empirique, issu de la pratique des entreprises, mais il présente également sur le plan scientifique, un caractère hybride si l'on considère les champs disciplinaires qui s'y sont intéressés. En effet, Les travaux de Damien Bruté de Rémur et Didier Lucas (Hardy 2009-2010), mais aussi ceux de Christian Marcon (Marcon 2012), de Philippe Dumas, François Lacroux, et Denis Gasté (Dumas, Lacroux, Gasté 2001) révèlent que le cœur de ce concept se situe au sein de couple SIC/sciences de gestion. Mais on le trouve également étudié par les sciences économiques, les sciences politiques, juridiques, la psychologie, la sociologie et la philosophie. Cette interdisciplinarité conduit même, du point de vue de certains spécialistes, à « un éclatement de l'objet » (Moinet 2012).

Nombre de problématiques de l'IE sont assujetties à l'hétérogénéité de ce concept et nous nous limiterons ici à celles qui se rattachent à notre discussion. Elles sont néanmoins centrales. D'un point de vue économique, les problématiques de l'IE oscillent entre les résistances que rencontre cette stratégie à s'installer dans les pratiques des entreprises et les difficultés à établir une convergence d'intérêt entre la sphère privée et la sphère publique. Il s'avère, par ailleurs, que le concept soit davantage établi dans les grandes entreprises et les ETI que dans les petites entreprises. La problématique des freins et des échecs d'instauration de cette stratégie dans les entreprises (Larivet 2002) perdure, même s'il apparaît aujourd'hui un déficit d'étude en faveur des plus petites entreprises (Guilhon 2004), voire une quasi-inexistence pour les TPE. Il a été admis à ce sujet, « une faible percée du discours sur l'IE dans les TPE françaises» (Frion 2012). Surgit alors un paradoxe, les petites entreprises seraient ignorées alors qu'elles représentent la grande majorité des entreprises. Seraient-elles les « grandes oubliées » de l'IE ?

Sur le plan scientifique, la problématique centrale se situe autour de l'appropriation de cet objet par diverses disciplines scientifiques ce qui contribue à compliquer l'édification de l'IE en un champ disciplinaire académiquement reconnu. Si les SIC sont devenues la discipline dominante au regard des publications scientifiques, thèses comprises (Marcon 2014), les sciences de gestion ont été les premières à s'y intéresser (Coggia 2009) en raison des liens entre l'IE et la prise de décision. Plusieurs autres disciplines 
s'en sont emparées diluant ainsi l'objet et rendant épineux l'établissement d'une reconnaissance de ce concept. Les fondements théoriques, les objets et méthodes sont hybrides et empiriques, accentuant ainsi les difficultés de mutation de ce concept en discipline.

Au sein des SIC, l'aspect informationnel a largement dominéles recherches jusqu'à présent (Marcon 2015) et l'aspect communicationnel semble délaissé, créant une situation inconfortable pour les publications et l'assise de ce concept en un objet scientifique solide. Les scientifiques rencontrent des difficultés à paraître dans des revues qualifiantes. En effet, d'une part, il n'existe pas de revue qualifiante en IE et d'autre part les revues classées en communication accueillent des articles « qui explorent essentiellement des questions communicationnelles alors que les auteurs abordent l'IE sous l'angle informationnel » (Marcon 2015). N'y a-t-il pas là un paradoxe ? Ceci amène plusieurs auteurs dominants (Moinet 2009), (Marcon 2014) à recommander d'orienter la recherche vers des sujets de communication afin de poursuivre la construction de l'IE en champ disciplinaire. La communication serait « une dimension oubliée de la communication ${ }^{3}$ ".

Cette contribution a pour ambition de renouveler les paradigmes sur l'IE en proposant une analyse communicationnelle de ce concept, tout en soumettant à la réflexion des postulats nouveaux à prendre en compte pour réédifier une intelligence économique adaptée aux petites et très petites entreprises.

Sur le plan épistémologique et théorique, nous nous positionnons dans le cadre des SIC. Nous adoptons une approche compréhensive et constructiviste, qui se situe dans l'héritage de l'école de Palo Alto, selon laquelle les acteurs et les composantes d'un espace de médiation s'inscrivent dans une démarche réflexive de co-construction d'une réalité sociale et économique. L'intelligence économique est un objet de recherche transdisciplinaire, mais il s'établit au cœur des SIC.

Il s'agit donc d'alimenter la discussion théorique et scientifique sur l'intérêt d'une approche communicationnelle et d'apporter des éléments nouveaux au débat afin d'enrichir le trop faible nombre d'études réalisées sur le sujet des TPE en SIC. Le corpus d'enquête présentera les difficultés d'appropriation du concept par les petites entreprises malgré les efforts des politiques publiques successives. En réalité, cet aspect du problème a été étudié au moins une fois et fait état, dans les petites entreprises, «d'un comportement informationnel de type intelligence économique émergent, auto-formé, une réponse intuitive, non-formelle et non-structurée que les informacteurs ont développé en réaction aux changements secouant l'environnement informationnel» (Mallowan 2014). Nous nous intéresserons aux petites entreprises en secteur innovant car on

3 Le n ${ }^{\circ} 42$ (2012) de la revue Communication \& Organisation, coordonnée par Thierry Libaert et Nicolas Moinet est entièrement consacré à l'intelligence économique sous le titre "La communication, dimension oubliée de l'intelligence économique ». Ils ont reçu, pour cet ouvrage collectif, le prix IEC 2013. 
peut supposer qu'elles se sont saisies de ces problématiques avant les autres, en raison de leurs activités créatives.

Dans ce cadre, nous avançons que l'intelligence économique dans la petite entreprise est fondée sur une série d'éléments qui sont contraires aux modélisations classiques de la discipline, que ce soit d'un point de vue professionnel ou d'un point de vie scientifique. Cette situation tient essentiellement aux contraintes spécifiques qui pèsent sur ce type d'entreprise. Cette hypothèse sera déclinée en trois axes majeurs : l'intelligence économique dans la petite entreprise en secteur innovant trouve sa source dans une culture collaborative de partage des informations. Cette culture collaborative de l'information est à la source de la construction des connaissances et savoirs mais aussi à l'origine de l'accès aux ressources nécessaires au chef d'entreprise dans l'établissement de sa stratégie. Enfin, l'acculturation à l'IE se met en ouvre dans un espace de médiation, que nous proposons d'appeler biocénose économique (que nous développerons plus bas) qui se compose d'un enchevêtrement d'interactions et d'interrelations entre plusieurs acteurs agencés en une superposition de réseaux. Ces acteurs sont les dirigeants des petites entreprises, mais aussi les structures interorganisationnelles de type semi étatiques dont la raison dêtre commune est le soutien au développement économique des petites entreprises et à l'innovation.

Afin de vérifier nos postulats de départ, nous avons mené une série de 17 entretiens avec des dirigeants de petites entreprises de moins de 50 salariés, toutes sous-traitantes du secteur aéronautique spatial défense en Nouvelle Aquitaine. Nous avons choisi d'explorer ce terrain car, jusqu'à présent, très peu d'études ont été réalisées bien que les petites entreprises fassent l'objet de plusieurs programmes en IE. Nous nous heurtons ici à un second paradoxe. Par ailleurs, les quelques études réalisées soulignent toutes les difficultés d'implémentation de l'IE dans ce type de structure. Nous rencontrons donc un double intérêt pour ce terrain.

Ces entretiens semi-directifs ont été guidés par l'utilisation d'un questionnaire. Ce questionnaire a fait l'objet pour sa construction d'une synthétisation des problématiques et postulats en vigueur dans la littérature professionnelle et scientifique sur l'intelligence économique, à laquelle s'ajoute une partie réservée à la politique publique d'IE.

Lexploitation du corpus a été opérée grâce à une méthode qualitative d'analyse de contenu par thématisation.La méthode qualitatives'est rapidement présentée comme une évidence au regard de la méthode de constitution du corpus et des objets recherchés. Les notions de culture informationnelle, d'élaboration de stratégie et les processus communicationnels recherchés semblent difficilement décelables à partir d'une étude quantitative. Les thèmes ont été identifiés à partir des hypothèses de départ et des postulats sur l'intelligence économique. Chaque thème a fait l'objet d'un travail de catégorisation (classification et codification des éléments relatifs aux 
hypothèses et postulats) afin de déceler des indices de présence ou d'absence. Une fois le corpus codifié, une synthèse dénombrant et quantifiant les thèmes a permis de procéder à une analyse inductive, confirmant ou infirmant les hypothèses et postulats de départ.

\section{Résultats}

Les dirigeants interrogés ont des comportements d'IE largement intuitifs et peu « appris » (17 dirigeants). Leurs pratiques sont largement fondées sur une culture informationnelle collaborative, faite d'échanges et de partage d'informations $\left(20^{4}\right)$, d'interrelations avec les contacts du dirigeant (19), d'exploitation d'un réseau identifié (16).

La majorité des dirigeants met en place une stratégie d'acquisition des savoirs et connaissances qui passe par l'action, la participation à des projets (13) mais aussi et surtout par des interactions avec les partenaires de l'environnement (17).

La majorité des entreprises se situe dans la technopole Bordeaux Technowest (15). Ils rencontrent des difficultés à définir avec précision la notion d'intelligence économique (15) qu'ils assimilent à la veille. Ils sont cependant engagés dans des actions stratégiques qui relèvent de l'IE : actions proactives dans un but concurrentiel (9), démarches de sécurité économique (4) ou actions d'influence de leur environnement (4).

Ils sont très peu à identifier les acteurs nationaux de l'IE (4) et les programmes dans ce domaine (9) mais ils discernent mieux les acteurs locaux (11).

À partir de ce moment, nous sommes amenés à considérer que la culture informationnelle collaborative est au cœur de l'intelligence économique dans les petites entreprises en secteur innovant. Il s'agit donc d'un processus communicationnel, ce qui va à l'encontre des modélisations conceptuelles académiquement reconnues sur le sujet, qui sont fondées sur des processus à dominante informationnelle. Ces petites entreprises développent une culture collaborative de l'information qui leur permet d'accéder aux ressources nécessaires à l'élaboration de leur stratégie et à la gestion quotidienne de leur entreprise. En effet, du fait des contraintes (structurelles, humaines et financières) auxquelles elles sont confrontées, elles n'ont pas les moyens, matériels et humains, d'accéder à ces ressources. Et pourtant, elles parviennent à conquérir des marchés, parfois de niche, à développer des innovations, parfois disruptives, tant au niveau local que national ou international. C'est cette culture collaborative de l'information qui les conduit à construire les connaissances, les savoirs, les savoir-faire nécessaires à leur existence ou à leur maintien sur un marché multipolaire et mouvant sur lequel l'innovation est cruciale. Ce passage à l'intelligence économique est le résultat d'un

4 Certains chiffres sont supérieurs à 17 car nous avons retenu dans nos règles d'énumération, l'intensité de la réponse, ce qui s'est traduit par une augmentation de la fréquence d'apparition des concepts clés dans le corpus. 
processus qui se réalise dans un cadre socio-économique original et propre à l'environnement de ces petites entreprises. Nous proposons à la discussion la notion de biocénose économique comme espace socio-économique géographiquement et économiquement situé. Nous montrerons que ses caractéristiques sont constitutives et conditionnelles à la mise en œuvre de l'intelligence économique par la petite entreprise en secteur innovant. $\mathrm{Ce}$ cadre va se substituer aux processus classiques de l'IE observés dans les grandes entreprises et modélisés pour elles : expression des besoins, collecte des informations, traitement et diffusion de l'information. Il met en ouvre des processus quasi inverses qui priorisent l'échange (diffusion) et le partage de l'information, l'action. Lors de cette phase, une prise de conscience du sens stratégique s'effectue et aboutit à la construction de savoirs, pratiques, stratégies.

\section{Vers une culture collaborative de l'information}

\section{Intelligence économique et culture collaborative de l'information}

Lintelligence économique des petites entreprises est fondée sur une culture informationnelle collaborative des acteurs qui la mettent en ouvre. Cette culture informationnelle nous semble indispensable à l'intelligence économique de ce type de structures et elle en constitue le cœur de la démarche. " $\mathrm{La}$ culture de l'information est proche de l'intelligence économique et territoriale dans le sens culture de l'information d'entreprise. En milieu organisationnel, il est possible de parler plutôt "d'intelligence informationnelle" vue comme une "intelligence collective organisationnelle" où plusieurs cultures (du texte, de l'hypertexte, des médias, des réseaux) sont mises à profit pour atteindre les objectifs de l'organisation » (Mallowan 2013). Ces compétences se retrouvent au cœur du concept d'intelligence économique (Bernat 2008) (Marcon 2011). L'intelligence de l'information est dédiée à l'aide à la prise de décision organisationnelle ». C'est grâce à cette culture que les acteurs, les dirigeants des petites entreprises vont " construire, organiser, classer les connaissances » (Liquète et Kovacs 2013) (Liquète 2014). Dans le cadre de notre recherche, nous nous inscrivons dans le schéma conceptuel de la culture informationnelle donné par Annette Béguin-Verbrugge et Susan Kavacs (2001) qui la définissent comme " un ensemble de connaissances et savoir-faire partagés dans une communauté (qui) permettent de situer, de repérer, de qualifier, de traiter et de communiquer des informations de manière pertinente » (Lehmans 2013). 
La mise en œuvre d'une culture collaborative de l'information

"Les pratiques de la culture collaborative de l'information, donnent une consistance symbolique et un système de formes et de représentation au lien social " (Lamizet 1992), ce qui se constitue le cœur de l'intelligence économique. Les dirigeants des petites entreprises vont faire circuler entre eux, entre pairs, dans un espace de communication dont nous dessinerons les contours dans la troisième partie de ce développement, des informations qui, en circulant, " assigneront un sens à la forme résultant du travail de l'information » (Lamizet 1992). Les petites entreprises rencontrent des difficultés à accéder aux ressources nécessaires à l'innovation, à la gestion de leur entreprise, à l'élaboration d'une stratégie. Elles doivent donc trouver des voies d'accès à la hauteur de leurs moyens.

Nous entendons par espace de communication, les espaces socioéconomiques thématiquement et géographiquement situés. Ceux-ci peuvent prendre la forme de diverses structures semi étatiques comme une pépinière d'entreprises, un cluster ou un pôle de compétitivité. La présence des dirigeants dans ces espaces de communication va les amener à entrer dans des processus de communication qui vont générer peu à peu une culture collaborative de communication. Ces processus de communication sont de nature interactionnelle, relationnelle. En effet, il s'agit des interactions physiques fondées sur le langage, le comportement verbal donc, mais aussi paraverbal, c'est-à-dire la conversation au sens large »(Vion 1999). Les relations par réseaux informatisés sont bien entendu présentes, mais elles ne sont pas déterminantes dans les processus.

Ces conversations vont leur permettre de constituer, développer un réseau, du fait du caractère thématiquement situé autour d'une activité économique définie, comme par exemple la technopole Bordeaux technowest, qui rassemble, à Mérignac, des entreprises du secteur aéronautique spatial défense. Cette technopole est divisée en plusieurs lieux, la pépinière dans laquelle s'installent les créateurs d'entreprise qui ont vocation à se développer, et le centre d'affaires dans lequel ils peuvent se déplacer une fois leur autonomie acquise. Cette technopole se situe au cœur de relations avec d'autres structures semi étatiques du même secteur d'activité ou de secteurs connexes, comme par exemple le cluster AETOS, sur les drones, ou le pôle de compétitivité en aéronautique spatial défense, Aerospace Valley. Il y a donc une communauté d'intérêts économiques entre les acteurs situés dans une même sphère. Cette communauté d'intérêts économiques met en œuvre des situations d'interactions et d'actions collectives comme les projets collaboratifs et collectifs des pôles de compétitivité ou des clusters, mais aussi des situations d'interrelations comme des salons professionnels, des conférences thématiques, des réunions d'information. On peut ainsi considérer que ces interrelations relèvent « de l'action située, qu'elles constituent des pratiques non formelles 
(qui) dépendent ainsi fortement du contexte dans lequel elles s'inscrivent et au sein duquel le sujet négocie son positionnement, son espace d'expression et d'appropriation. La sphère non formelle structure la relation à l'information » (Cordier 2013).

On assiste à un processus d'autonomisation des acteurs qui vont peu à peu " utiliser leurs propres capacités pour agir dans diverses situations et face à des environnements différents " (Liquète 2011). Le fait d'être inséré dans un collectif permet de partager avec les autres des questions stratégiques liées essentiellement à la réduction de l'incertitude, à l'asymétrie de l'information (Viviani 2016), à l'exercice de la conduite quotidienne de l'entreprise (gestion, finance, ressources humaines), mais aussi et surtout d'atteindre ou de repérer comment trouver des ressources techniques et concurrentielles difficilement accessibles par manque de moyens. Ce partage d'information est donc une manière de s'autonomiser, de se confronter, de se renforcer, de s'enrichir, de donner du sens à l'information.

Pour ces dirigeants, la coopération est la solution la plus efficace et la moins coûteuse. Il faut prendre en compte les spécificités de ce modèle d'échange et sa capacité à bien fonctionner dans des communautés de savoir. Une des raisons du succès de ces démarches serait que « les échanges de connaissances n'opèrent pas selon le même mode que l'échange de biens. Quand on échange une connaissance, une information, on ne la perd pas. [...] Autre argument à prendre en compte, l'acquisition comme la production de connaissances complexes serait un processus intrinsèquement coopératif où chaque agent dépendrait d'autrui pour produire "(Conein 2004) (Callon 1986). Ce phénomène explique la non rivalité des relations entre acteurs et le fait que ces communautés existent sans relation hiérarchique, ni domination d'un des acteurs. Il s'agirait en quelque sorte d'une « utopie raisonnée » (Spreber 1999).

\section{De la culture collaborative de l'information à la construction de connaissances}

Cette culture informationnelle "est propre à la société de l'information ", selon Claude Baltz (1998) car « elle permet d'identifier les connaissances, une vision, des postures, des façons d'agir » (Lehmans 2013). C'est ainsi que les acteurs-entrepreneurs vont être peu à peu amenés à créer des connaissances, des savoirs dont ils ont besoin pour leur activité. Leur activité est essentiellement à caractère immatériel ou fondée sur l'utilisation de ressources immatérielles. Or ces créations de connaissances sont possibles lorsque se constituent des communautés d'apprentissage qui vont donner naissance à une intelligence collective. Cette dernière est la composante essentielle de l'intelligence économique. 


\section{Les communautés d'apprentissage}

Les interactions des dirigeants de petites entreprises dans les espaces socioéconomiques vont les conduire à créer des sortes de collectifs, des réseaux. Ces interrelations peuvent d'ailleurs se situer à un niveau réticulaire plus complexe et à un stade supérieur à une seule organisation. Elles ont lieu entre plusieurs structures semi étatiques rassemblées autour d'activités économiques proches et qui seront amenées à s'entrecroiser à certaines occasions, comme des salons professionnels thématiques. Il s'agit donc d'un enchevêtrement de réseaux locaux entre les divers acteurs : les entreprises et les structures semi étatiques. Ces collectifs s'approchent de la notion bien connue de communauté de pratique (Wenger 2005) et sont en fait des communautés d'apprentissage. L'apprentissage est possible car, du fait du processus de culture collaborative d'information, les acteurs ont acquis des langages communs, des expériences communes qui peuvent être comprises et interprétées parles autres. En effet, des structures comme les pépinières, clusters et pôles de compétitivité entrainent les acteurs à travailler ensemble autour de projets financés à la condition de rassembler, autour d'un thème donné, des organisations de nature et de taille différentes : les PME, les grandes entreprises, les laboratoires de recherche. Dans ces actions communes, ces travaux partagés, ils développent une culture commune souvent technique, mais ils partagent aussi des connaissances, des méthodes, des process, " une façon de penser et catégoriser l'information selon un modèle qui fait consensus (Stadler 2013). «Les interactions résultent du contexte d'apparition et des pratiques sociales d'interaction en réseaux. Elles sont ancrées dans une culture qui leur donne du sens [...]" (Bruner 1996). Cela rejoint les études de Lam et d'autres qui montrent comment les modes d'apprentissage et d'innovation dans la Silicon Valley, à Cambridge, au Japon ou au Danemark, varient en fonction des facteurs institutionnels tels que les communautés professionnelles ou les systèmes d'enseignement » (Delamotte 2004). On retrouve dans ces structures les quatre caractéristiques de la communauté d'apprentissage selon Dillenbourg, Poirier et Carles (Dillembourg 2003) : les échanges sont faits sous forme narrative, l'inférence est favorisée par une culture commune, la co-construction de l'expérience personnelle et l'apprentissage sont fortement situés et contextualisés. Dans ces communautés d'apprentissage, on peut considérer que les acteurs apprennent dans l'interaction si l'on se réfère aux travaux de Mina Verba qui postule " qu'il y a apprentissage dans l'action si on repère une activité cognitive, la reproduction des actions (qui existe bien dans les communautés autour des projets communs mis en œuvre), s'il y a partage d'intérêt vers le partenaire et son activité, (ce qui est effectivement le cas dans les collectifs décrits) et si l'on constate une gestion autonome, c'est-à-dire un engagement des acteurs, 
des prises d'information par l'observation, une gestion sociale, du feed back » (Verba 1999). Ces communautés d'apprentissage sont le lieu de l'intelligence collective.

L'intelligence collective

La culture de l'intelligence n'est pas acquise est reste difficile à mettre en œuvre. C'est un des enjeux de l'intelligence économique (Moinet 2008). Pour Doise, Mugny \& Perret-Clermont, et Carugaty, « la genèse de l'intelligence relève de l'interaction dans laquelle le "conflit" joue un grand rôle. L'interaction avec autrui fournit des informations qui aident dans l'élaboration d'une nouvelle réponse, ce qui se produit notamment dans les couples avec des niveaux de connaissance différents, surtout lorsqu'un des deux partenaires est capable de fournir des directives opératoires " (Agostinelli 2000). Sur la base des paradigmes marshalliens, il apparaît que les interrelations développées par les acteurs au sein des communautés d'apprentissage sont à la source de l'intelligence économique. Du fait de la notion de communauté, on peut parler d'intelligence collective. Au sein de ces communautés, les interrelations sont des échanges d'informations, nous l'avons vu, mais aussi des travaux collectifs qui relèvent de la négociation (Aballea 2003), du conflit, au sens non guerrier du terme. Il s'agit d'une négociation désintéressée dans le sens ou la notion de pouvoir est absente. Cette procédure de négociation à un niveau collectif est le moteur de l'apprentissage au sens vygotskien du processus de construction des connaissances. "Ce sont les relations interindividuelles qui conduisent à l'apprentissage ». Ceci a d'ailleurs été mis en évidence par " les travaux de Sanexian qui montre, au sujet de la Silicon Valley, que la communication interpersonnelle joue un rôle considérable dans la circulation des connaissances entre les entreprises et entre les secteurs industriels " (Delamotte 2004). On peut ainsi voir les activités interactionnelles des acteurs comme des processus collaboratifs qui conduisent à l'apprentissage. Du fait que cet apprentissage s'effectue collectivement, on peut considérer que les acteurs développent une intelligence collective.

\section{La biocénose économique en tant qu'espace de médiation et d'acculturation à l'intelligence économique.}

La biocénose économique, est un emprunt allégorique au concept développé en écologie et en biogéographie biologique, transféré au domaine économique, en vue d'identifier, comprendre, analyser les processus d'intelligence économique, la création de savoirs et connaissances des petites entreprises en secteur innovant.

La biocénose est définie comme "l'ensemble du peuplement vivant en une aire donnée » (Lacoste 2009). L'apport fructueux de cette notion est son caractère syncrétique "fondé sur la coexistence au sein d'un même biotope, 
de diverses communautés végétales et animales entre lesquelles se trouve développé un réseau complexe d'interactions » (Lacoste 2009). « La biocénose se caractérise par des relations intra spécifiques, c'est-à-dire des relations qui s'établissent au sein de la même espèce et des relations interspécifiques, c'est-à-dire entre des espèces différentes " (Lamy 2001), pour conduire à une correspondance étroite entre ses composantes. Ainsi dans un biotope donné, du fait des relations interspécifiques et intraspécifiques, certaines espèces se développent en association avec d'autres qui leurs sont spécifiques.

Ramené au domaine économique, on pourrait avancer que les différentes catégories d'acteurs associées géographiquement et économiquement dans une sphère économiquement et socialement située, développent des relations intra spécifiques et interspécifiques qui conduisent à la création d'un espace, de relations et d'actions qui leurs sont spécifiques.

En recherchant les fondements du développement de l'intelligence économique dans les petites entreprises, nous considérons que ce concept favoriserait l'édification d'un modèle d'intelligence économique propre aux petites entreprises en secteur innovant. Il permettrait, selon nous, une mobilisation des savoirs (Brassac 2008) [qui seraient] à la fois une connaissance - objet sur la base « des modèles pédagogiques transmissifs ou behaviouristes, dans lesquels les connaissances seraient identifiées, classifiées et à terme programmables ». Par ailleurs, cet espace serait également et surtout le lieu de la connaissance - incarnée mise en œuvre par « des praxis, des distanciations cognitives à travers des retours d'expérience, mais aussi des conflits cognitifs, à travers des activités de débats ", des conversations entre acteurs installés dans l'espace économique. «La subjectivité des acteurs ainsi que les constructions intersubjectives, qui prennent la forme d'allant de soi ou d'ethnométhodes partagées par les membres de la communauté cognitive formée au cours de ces espaces d'interactions, constituent le cœur des démarches constructivistes " (Kiyindou 2011) de notre modèle d'intelligence économique dans la petite entreprise innovante.

Nous allons ici tenter de caractériser cet espace qui revêt une triple dimension : c'est une sphère située géographiquement et économiquement autour d'un secteur d'activité déterminé. On retrouve ici la notion de proximité spatiale (Grosseti 2008) qui montre l'importance considérable des relations personnelles dans toutes les situations d'accès à des ressources externes comme cela a été montré au sujet de la création d'entreprise.

Cet univers relève des réseaux dans son modèle de fonctionnement et les acteurs en interactions sont assemblés par des relations relevant des réseaux au sens large. La particularité de cette sphère est que l'enchevêtrement des réseaux qui la caractérise devient un instrument d'allocation des ressources. Aucune hiérarchie, pas de lien fonctionnel, uniquement des interrelations désirées, puisées dans un sens donné par l'action. 
Enfin l'ensemble des interactions entre les acteurs, qualifiées de relations intra spécifiques (à l'intérieur de chaque catégorie d'acteur) et de relations inter spécifiques (entre les différentes catégories d'acteurs) participe à la construction d'un espace collaboratif de création et de socialisation de connaissance. C'est au sein de ce domaine que sera mise en œuvre la démarche d'intelligence économique des petites entreprises en secteur innovant. Elle doit être vue comme un cadre de dynamisme des phénomènes sociaux.

\section{Comment caractériser les acteurs de la biocénose économique?}

La biocénose économique se compose de l'ensemble des relations, interrelations, physiques mais aussi numériques entre plusieurs acteurs. Ces acteurs sont les dirigeants des petites entreprises qui sont installés dans des structures institutionnelles semi-étatiques que nous qualifierons d'interorganisationnelles. Ces structures, plus ou moins directement financées par l'État ou une collectivité locale, sont des associations, des pépinières, des technopoles, des clusters, des pôles de compétitivité, ayant pour vocation le soutien institutionnel au développement économique et à l'innovation. Toute structure relative au district industriel décrit par Marshall. Ces dernières sont également des acteurs de la biocénose car elles insufflent des actions qui vont favoriser les interactions, des réciprocités, l'échange etle partage d'informations, des actions communes. L'ensemble présente la forme d'un enchevêtrement de relations complexes dont les limites ne sont pas définies mais qui installent un sentiment d'appartenance et de proximité proche de l'organisation. À ce titre, on pourrait qualifier l'ensemble de quasi-organisation (Bernoux 2010). Ces structures établies sur une logique communicationnelle associée à une stratégie réseau, ne relèvent pas des formes structurelles classiques mais ont tendance à être " adhocratiques " au sens de Mintzberg, c'est-à-dire qu'elles se composent d'un ensemble de relations variables et mouvantes, informelles, dont la densité crée un état proche de la structure organisationnelle sans en être une. La communication est donc le " processus organisant " de la biocénose économique. On retrouve dans cette quasi-organisation, trois formes de proximité, géographique, institutionnelle et organisationnelle qui « constituent trois piliers de l'action collective » (Talbot 2008).

Cet espace doit être considéré comme un « médium qui rend possible la communication entre les interlocuteurs » (Peraya 2010) car,comme le considère Dominique Volton, « l'information est toujours et systématiquement liée aux valeurs et à des intérêts situés dans des aires culturelles qui ne lui donnent pas la même signification " (Liquète 2014). L'intelligence économique de la petite entreprise prend sa source dans cet espace car il donne lieu à la contextualisation des informations et par conséquent donne du sens aux informations et ressources échangées. À ce titre, il peut être considéré comme un espace de médiation. 


\section{Un espace de contextualisation des informations}

La notion de contexte nous semble déterminante dans le processus d'intelligence économique. C'est parce que les acteurs économiques sont mis dans une situation de contexte particulière que la communication va pouvoir sétablir et peu à peu créer un référent culturel commun constitutif du terreau de la culture collaborative de l'information et donc de l'intelligence économique. Ces acteurs sont issus de contextes différents, ce qui ne leur permet pas d'établir une communication efficace en raison des composantes hétérogènes de la communication. Les variables décrivant le contexte d'une situation (Argentin 1989) apparaissent dans la biocénose économique. Cette dernière met en œuvre des canaux de communication ouverts - successivement ou simultanément - des éléments temporels, des lieux permettant de déterminer la position et l'espace/distance séparant les protagonistes, une description $\mathrm{du} / \mathrm{des}$ locuteurs et/ou auditeurs aussi bien que des non participants s'il y en $\mathrm{a}$, des conditions de l'environnement (lumière, espace, bruit/silence, dimensions). Tous ces éléments ordonnent un contexte spécifique qui va permettre d'agencer la circulation et l'établissement d'une communication au sein de l'espace considéré. En partageant cet espace commun, un contexte commun va se créer et c'est ce qui fera la différence dans le succès ou l'échec de l'établissement d'une culture collaborative et du processus d'intelligence économique pour les petites entreprises. C'est souvent le contexte qui fait toute la différence entre succès et échec (Morgan 2000) et l'absence d'étude du contexte est souvent à la racine des difficultés à expliquer les difficultés communicationnelles.

$\mathrm{Au}$-delà d'une culture commune, le contexte va permettre de donner du sens aux informations échangées. Il va en quelque sorte se substituer aux structures organisationnelles traditionnelles. La notion de quasi-organisation parait adaptée à cette situation des acteurs. Comme s'ils étaient rassemblés au sein de la même organisation, leur communication va être perçue comme un processus d'interactions et de significations partagées, ce que Weick appelle le sensemaking (Weick 1969). Ce processus va permettre aux acteurs de construire, filtrer, structurer et ainsi transformer le subjectif en quelque chose de plus tangible (Weick 2001 ; MacIntosh-Murray 2005). La création de sens doit permettre de faire face à l'environnement. Si l'intelligence économique est une stratégie permettant de positionner l'entreprise dans son environnement et d'avoir une approche globale et systémique, les stratégies d'IE, en tant qu'objet, n'existent et ne prennent sens que par rapport aux intentions des acteurs, insérés dans un système global. Pertinence, construction mentale (Watzlavick 1972), intention, ces éléments se mettent en œuvre dans le processus relationnel d'interactions permanentes et continues entre les acteurs de la stratégie d'intelligence économique, au-delà du simple recueil des informations. Les acteurs, en réalisant leurs interactions, se " découplent » 
et « finissent par prendre conscience qu'ils constituent une sorte de collectif. Le passage de la forme de réseau à celle du collectif s'effectue par la création de ressources de médiations spécifiques » (Grossetti 2010). Cela se transforme peu à peu en un processus d'apprentissage : learning by doing, by using, learning to learn (Camagni 2006).

\section{Une espace de médiation}

La médiation a pour fonction et pour résultat de transformer la relation entre les sujets (les acteurs) ou entre les sujets et des objets (des savoirs, des connaissances) (Peraya 2010). «La médiation représente l'impératif social majeur de la dialectique entre le singulier et le collectif " (Lamizet 1999). À ce titre, les espaces socio-économiques comme la biocénose économique représentent le creuset de l'intelligence économique. Il s'agit du " lieu » métaphorique où les relations entre acteurs vont se transformer, où ils vont construire cette culture collaborative dont nous parlons, où ils vont accéder à des ressources, créer des connaissances. Ce sont des espaces de médiation au sens de Lamizet : " un espace social régulé non par la seule intervention d'acteurs institutionnels, mais aussi par la diffusion des représentations de la médiation assurée par ces acteurs et de leurs stratégies " (Lamizet 1992). C'est dans ces espaces que le lien va être fait entre le singulier, le local c'est-à-dire, le quotidien de l'acteur isolé dans son organisation avec des problématiques et le collectif, le global, c'est-à-dire le secteur d'activité avec ses marchés, ses réglementations et normalisations internationales. Ces espaces établissent le lien entre le local et le global, dans la mesure où le local communique au global les éléments qu'il perçoit et le global les positionne dans leur contexte.

Ces interactions favorisent des stratégies interactionnelles de nature informationnelle mais aussi et surtout communicationnelle. La part de la communication domine clairement dans ces stratégies, ce qui nous amène à affirmer que l'intelligence économique dans la petite entreprise présente un aspect original et non reproductible qui se situe à l'inverse des modélisations classiques de l'intelligence économiques. En effet, rappelons que les modèles classiques dominants (Levet \& Paturel 1996 ; Béguin, Deschamps et Madinier 2007 ; Faivre 1991 ; Bulinge 2013) placent tous l'information ou le savoir au commencement des cycles représentés. Ce sont des cycles à dominante informationnelle.

Le sens naît dans ces lieux et usages en transformant des pratiques communicationnelles comme les actes de langages et les actions singulières d'un acteur en procédés et actes de langage collectifs structurés autour de thématiques économiques définies par le secteur d'activité. Il s'agit d'une co-construction d'une culture dans la réalité qui ne peut avoir lieu dans les espaces isolés que sont les bureaux indépendants des entreprises, ni dans des zones économiques qui ne font l'objet d'aucun aménagement physique et 
stratégique de l'espace, sans le réseau d'organisations institutionnelles dont le rôle est le soutien au développement économique. Nous insistons ici, sur le caractère réticulaire de l'espace décrit. Ce n'est pas non plus la seule présence d'un ou plusieurs acteurs qui est déterminante, c'est la mise en réseau des divers acteurs organisée en une sorte de millefeuille.

Ces lieux créent des « espaces symboliques » (Lamizet 1992) dans lesquels vont se constituer les territoires économiques des acteurs, ce qui représente un morcellement de l'espace social global. Ceci nous amène à comprendre l'importance du rôle de ces structures institutionnelles dans la création d'une dynamique pour les acteurs qui vont y construire une stratégie pour leur entreprise et y trouver les ressources nécessaires. Dans la biocénose, on va retrouver trois des quatre figures emblématiques de l'autre dans la construction géographique de l'espace symbolique : l'interlocuteur, le voisin, le traité (un espace de convention, de partage du marché entre deux partenaires de communication). Cet aspect géométrique doit être vu, non pas comme une réalité physique, mais comme la réalité de sa perception par l'acteur, qui ainsi, en fait un espace pensé.

La transition entre l'entrée d'un acteur dans la quasi-organisation et l'appropriation d'une culture collaborative de l'information va s'effecteur par l'entremise de deux facteurs qui vont faire que cet espace va devenir un objet de savoir. Ces deux facteurs sont la mémoire de l'espace et le caractère géographiquement situé de l'espace. Cette quasi-organisation devient alors " un espace reconnu » par l'acteur, un espace pertinent (Marcon 2014) et il va pouvoir mettre en œuvre ses stratégies intellectuelles, d'apprentissage, d'appropriation des ressources économiques, sa stratégie d'intelligence économique.

La biocénose économique instaure donc une correspondance étroite entre les acteurs au sein de cet espace métaphorique. Il en résulte le déclenchement d'un processus d'intelligence économique spécifique aux petites entreprises en secteur innovant du fait des difficultés à mettre en ouvre les modèles instaurés et préconisés par la doxa. Les contraintes structurelles de cette catégorie d'entreprise n'enrayent pas leur capacité à se positionner dans leur environnement au sens de l'intelligence économique. Elles en changent les modalités. Par des démarches essentiellement communicationnelles, elles vont développer un modèle original centré sur l'accès aux ressources par l'action et l'expérience.

Cette réflexion remet au centre des discussions le cycle du renseignement et la démarche stratégique des acteurs dans un contexte donné. Elle propose de reconsidérer à un niveau global, le rôle des institutions dans cette stratégie et plus précisément les modalités de mise en œuvre des politiques publiques d'intelligence économique à destination des petites entreprises. Elle élargit le champ paradigmatique de la discipline en offrant un angle d'analyse communicationnel jusque-là peu développé et un terrain d'étude 
qui participera au processus de construction de l'intelligence économique en tant qu'objet scientifique. Confortant le caractère interdisciplinaire voire transdisciplinaire de la matière, elle propose de croiser les théories afin de construire un appareillage permettant d'élargir l'approche conceptuelle et de l'enrichir.

\section{BIBLIOGRAPHIE}

ABALLEA F. B., 2003, « Réseaux et innovations organisationnelles. Une approche par les relations professionnelles », Travail et emploi, 95, 88.

AGOSTINELLI S. M., 2000, Espaces communs de communication des connaissances : E3C, Bordeaux, Presses Universitaires de Bordeaux.

ARGENTIN G., 1989, Quand faire, c'est dire, Liège, Pierre Mardaga.

BATAZZI-ALEXIS C., 2002, Les technologies de l'information et de la communication (TIC) dans un processus d'apprentissage organisationnel: pour une coordination émergente entre le local et le global, Communication et organisation, 22.

BERNAT J. P., 2008, «Lintelligence économique: co-construction et émergence d'une discipline via un réseau humain ", Hermès, 141-173.

BERNOUX P. 2010, La sociologie des organisations, Paris, Éditions du Seuil.

BRASSAC C., 2008, «L'acquisition de savoirs comme activité située et distribuée »,

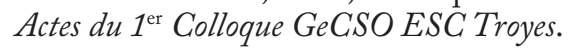

BRUNER J., 1996, L'éducation, entrée dans la culture, Paris, Retz.

CALLON M.,1986, «Élements pour une sociologie de la traduction : la domestication des coquilles Saint-Jacques et des marins-pécheurs de la baie de Saint Brieuc ", L'année sociologique, 169-208.

CAMAGNI R. M., 2006, Milieux innovateurs, Paris, Economica.

CARAYON B., 2003, Intelligence économique, compétitivité et cohésion sociale, La Documentation française, collection des rapports officiels.

COGGIA V., 2009, Intelligence économique et prise de décision dans la PME: le défi de l'adaptation des procédés de l'intelligence économique aux particularités culturelles des petites entreprises, Paris, L'Harmattan.

CONEIN B., 2004, «Les communautés informatiques comme communauté épistemique», dans E. DELAMOTTE, Du partage au marché, Villeneuve d'ascq, Presses Universitaires du Septentrion, 57-76.

CORDIER A. L., 2013, "La culture générale face à l'information», Cultures de l'information, 69-89.

DELAMOTTE E., 2004, « De l'économie de la connaissance à la société de la connaissance ", dans E. DELAMOTTE, Du partage au marché, Villeneuve d'Ascq, Presses Universitaires du Septentrion, 9-38. 
DILLEMBOURG P.P., POIRIER C., CARLES L., 2003, « Communautés virtuelles d'apprentissage : e-jargon ou nouveau paradigme ", Pédagogies. Net. Montréal, Presses, 11-47.

DORTIER J., 2016, La communication, Paris, Sciences humaines éditions.

DUMAS P., LACROUX F., GASTÉ D., 2001, « Décider ensemble: Complexité des apprentissages et convergence des disciplines », Colloque Communication et Entreprise.

FRION P., 2012, Généalogie de la faible percée du discours sur l'intelligence économique dans les TPE françaises : errements épistémologiques et propositions opérationnelles, thèse de Doctorat, Poitier, ACRIE.

GROSSETIM., 2008, « Réseaux sociaux et médiations dans les activtés d'innovation », Communiquer - Innover HERMES, vol. 50, 21-27.

GROSSETI M., 2010, « Réseaux sociaux et ressources de médiation», Médiations, 103-120.

GUILHON A., 2004, L'intelligence économique dans la PME, visions éparses, paradoxes et manifestations, Paris, L'harmattan.

GUYOT B., 2002, « Mettre en ordre les activités d'information, nouvelle forme de rationalisation organisationnnelle ", Enjeux de l'information et de la communication, 49-64.

HARDY M., 2011, Le concept français d'intelligence économique : histoire et tendances, Shanghai, Shanghai Renmin Chubanshe.

KIYINDOU A., 2011, Nouveaux espaces de partage des savoirs, Paris, L'Harmattan.

LACOSTE A. S., 2009, Élements de biogéographie et d'écologie, Paris, Armand Colin.

LAMIZET B., 1992, Les lieux de communication, Liège, Mardaga.

LAMIZET B., 1999, La médiation culturelle, Paris, L'Harmattan.

LAMY M., 2001, L'écologie humaine, Paris, Ellipses.

LARIVET S., 2002, Les réalités de l'intelligence économique en PME, Université du Sud Toulon-Var. UFR de Sciences économiques et de gestion.

LEHMANS A., 2013, «La culture de l'information: ruptures et invariants épistémologiques ", dans V. LIQUĖTE (dir.), Cultures de l'information, Paris, CNRS Editions, 31-51.

LIQUÈTE V., 2011, Des pratiques d'information à la construction de connaissances en contexte, Rouen, Université de Rouen.

LIQUĖTE V. 2014, “Présentation générale réflexion historique, culturelle et communicationnelle autour des cultures de l'information », dans V. LIQUĖTE, Cultures de l'information, Paris, CNRS Éditions, 9-30.

MALLOWAN M. 2013, "La transculture de l'information : vers une posture multicuturelle de l'information ", dans V. LIQUĖTE, Cultures de l'information, Paris, CNRS Éditions, 159-175.

MALLOWAN M. 2014, "De l'intelligence économique comme état d'esprit à la transculture de l'information ", in Revue internationale d'intelligence économique, 2, [25/01/2016], www.cairn.info/revue-internationale-d-intelligence-economique-20142-page-111.htm 
MARCON C. M., 2011, L’intelligence économique - 2e édition, Paris, DUNOD.

MARCON C., 2012, « De la mesure aux hypothèses explicatives, regard sur 15 ans d'oubli », Revue Communication et Organisation, 42, 13-26.

MARCON C., 2014, La recherche française en intelligence économique, Paris, L'Harmattan.

MARCON C. 2015, «Approche bibliométrique élémentaire des publications en intelligence économique dans les revues françaises en sciences de l'information et la communication », Communication et organisation, 47, 236-252.

MARTRE H., 1994, Intelligence économique et stratégie des entreprises, Paris, La Documentation Française. Premier rapport étatique comportant le terme intelligence économique dans son titre.

MOINET N., 2008, « La maîtrise de l'information stratégique dans les organisations au regard du couple agilité/paralysie : la notion de dispositif intelligent. ", dans V. COUZINET, Linformation dans les organisations : dynamique et complexité, Tours, Presses universitaires François Rabelais, 67-81.

MOINET N., 2009, « Lépistémologie de l'intelligence économique face au défi de la communication. » Revue internationale d'intelligence économique, 1, 2, 159-173

MOINET N., 2011, Intelligence économique, Mythes et réalités. Paris, CNRS Éditions.

MOINET N., 2012, Petite histoire de l'intelligence économique, Paris, L'Harmattan.

MORGAN G., 2000, Les images de l'organisation, Paris, de Boeck.

PERAYA D., 2010, «Médiatisation et médiation : des médias éducatifs aux ENT », dans V. LIQUÈTE, Médiations, Paris, L’Harmattan, 33-48.

STADLER A. D., 2013, «Informer, s'informer en contexte professionnels : une approche par le document technique ", dans V. LIQUÈTE, Cultures de l'information, Paris, L'Harmattan, 91-113.

TALBOT D., 2008, « Les institutions créatrices de proximité. Institutions as creators of proximities », Revue d'Économie Régionale Ė Urbaine 3, 289-310.

VERBA M., 1999, « Dynamiques interactives dans la construction des savoirs », dans M. R. GILLY, Apprendre dans l'interaction, Nancy, Presses Univesitaires de Nancy, 181200.

VION R., 1999, « Linguistique et communication verbale », dans M. R. Gilly (Dir.), Apprendre dans l'interaction, Nancy, Presses Universitaires de Nancy, 41-67.

VIVIANI J. VO T., 2016, «Peut-on arréter de jouer au "maillon faible" dans la chaîne logistique ? Le financement collaboratif du BFR dans la chaîne logistique estil possible ? ", dans L. BIRONNEAU \& V. J. (Dir.), Collaboration et réseaux : approches transversales en management, Rennes, Presses Universitaires de Rennes, 151-170.

WATZLAVICK P. et al., 1972, Une logique de la communication.

WEICK K., 1969, The Social Psychology of Organizing, New York, Random House.

WENGER E., 2005, La théorie des communautés de pratique, Laval, Presses Université Laval. 
Résumé : L'intelligence économique est un concept récent qui présente l'originalité d'être à la fois une stratégie d'entreprise, un objet scientifique et une politique publique. Sur le plan scientifique, il se trouve au cœur de plusieurs disciplines dont les SIC et les sciences de gestion ce qui permet des approches interdisciplinaires, voire pluridisciplinaires. En tant que stratégie d'entreprise, le concept est assez bien implanté dans les grandes entreprises et les structures de taille moyenne. On observe cependant une résistance du concept auprès des petites et très petites entreprises. Cette contribution à pour objet de renouveler les paradigmes sur le sujet en proposant un angle d'analyse communicationnel de cet objet, qui jusqu'à présent a surtout fait l'objet d'études sous un angle informationnel. Ce faisant, il s'agira de proposer un modèle d'intelligence économique adapté à ce type de structures. Il apparait, en effet, que les petites entreprises mettent en œuvre des stratégies d'intelligence économique informelles, assez intuitives qui sont le résultat des contraintes qu'elles subissent du fait leurs particularités structurelles, organisationnelles et humaines. Les modèles classiques de l'intelligence économique conçus et établis avec succès dans les grandes entreprises, ne concernent que marginalement ces petites structures. Les stratégies d'intelligence économique de ces dernières mettent en œuvre un assemblage d'actions et tactiques originales, résolument pragmatiques. Leurs démarches reposent tout d'abord sur la construction d'une culture informationnelle collaborative des dirigeants. Cette culture de partage des informations va leur permettre d'accéder aux ressources nécessaires à la gestion de leur entreprise, notamment essentiellement des ressources intangibles, comme les connaissances et les savoirs. Ces processus spécifiques se forment dans un espace de médiation, la biocénose économique, qui se constitue d'un ensemble d'interactions et d'interrelations entre plusieurs acteurs dont les institutions semi-étatiques d'aide au développement des entreprises et à l'innovation. Cette sphère socio-économique dont nous allons exposer les caractéristiques est le lieu de déploiement de la culture informationnelle des dirigeants d'entreprise et celui de la création d'une intelligence économique de la petite entreprise.

Mots-clés : intelligence économique, culture informationnelle, médiation, PME,TPE, stratégie, intelligence collective.

Abstract: Competitive intelligence is a recent concept that presents the originality of being at the same time a strategy of enterprise, a scientific object and a public policy Scientifically, it is at the heart of several disciplines, including CIS and management sciences, which allows for interdisciplinary and even multidisciplinary approaches. As a business strategy, the concept is fairly well established in large companies and medium-sized structures. There is, however, a resistance of the concept to small and very small enterprises. This presentation aims to renew the paradigms on the subject by proposing an angle of communication analysis of this object, which up to now has been the object of studies from an information angle. In so doing, a competitive intelligence model adapted to this type of companies will be presented. Their competitive intelligence strategies involve an assemblage of original and resolutely pragmatic actions and tactics. It appears that small firms implement informal, rather intuitive, competitive intelligence strategies, that are the result of the constraints they face because of their structural, organizational and human characteristics. The classical models of economic intelligence designed and established successfully in large firms only marginally affect these small structures. The strategies of competitive intelligence in small corporate implement an assemblage of original actions and 
tactics, resolutely pragmatics. Their approaches are based first of all on the construction of a collaborative information culture of the leaders. This culture of information sharing will enable them to access the resources needed to manage their business, especially essentially immaterial resources such as knowledge and cognition. These specific processes are formed in a mediation space, economic biocenosis, which is constituted of a set of interactions and interrelations between several economic actors including state institutions for business development and innovation. This socioeconomic sphere, the characteristics of which will be described, is the place where the information culture of corporate leaders is deployed. It is the creation place of a competitive intelligence of small enterprises.

Keywords: Competitive intelligence, information culture, médiation, Small-and-MediumSized Entreprises, very small entreprises, strategy, collective intelligence. 